\title{
Massive pulmonary embolism with ST-elevation in the inferior leads and other interesting ECG findings
}

\author{
Said Alsidawi ${ }^{1}$, Mouhamad Abdalla ${ }^{2}$, Tarek Helmy ${ }^{2}$ \\ 1. Department of Internal Medicine, College of Medicine, University of Cincinnati, USA. 2. Division of Cardiovascular \\ Diseases, Department of Internal Medicine, College of Medicine, University of Cincinnati, USA
}

Correspondence: Said Alsidawi. Address: 2324 Madison Rd. 1003, Cincinnati, OH 45208. Email:

alsidasd@ucmail.uc.edu.

Received: June 19, 2012

Accepted: August 15, 2012 Online Published: December 17, 2012

DOI : $10.5430 /$ jbgc.v3n1p43

URL: http://dx.doi.org/10.5430/jbgc.v3n1p43

\section{Abstract}

Introduction: Pulmonary embolism is associated with many ECG findings, most of which are non-specific and most can be explained by the sudden severe increase in the right ventricular afterload leading to dysfunction, hypoperfusion, dilation and in rare very severe cases to ischemic injury.

Many case reports described patients presenting with massive pulmonary embolism and very rare atypical ECG findings especially ST-segment elevation in the anteroseptal leads (V1-V4).

Case presentation: We present a case of a 73-year old African American male who suffered from a massive pulmonary embolism with interesting ECG findings mainly ST-segment elevation in the inferior leads mimicking Inferior wall myocardial infarction.

To our knowledge, this is the first case of ST-elevation in the inferior leads in the setting of a massive PE.

Conclusion: The most likely explanation to the case is that the associated cardiac injury is multifactorial. Severe right ventricular dilation with significant increase in wall tension and oxygen consumption, sudden coronary hypoperfusion caused by the sudden drop in the right and left ventricular output, hypoxia caused by the massive PE and finally possible coronary spasm caused by hypoxia and increased right heart pressure might all have contributed to inducing the acute right ventricular ischemia which showed as ST-segment elevation in the inferior leads and an elevation in cardiac enzymes.

\section{Key words}

Pulmonary embolism, ST-elevation, Angiogram, Echocardiogram

\section{I ntroduction}

Pulmonary embolism is caused by an obstruction of the pulmonary artery or one of its branches, usually occurring when a deep vein thrombus becomes dislodged from its site of formation and embolizes, to the arterial blood supply of one of the lungs.

We present a case of massive pulmonary embolism with interesting ECG findings. To our knowledge, this is the first case to report such findings. 


\section{Case presentation}

This is a 73-year-old African American male with no known past medical history who had a witnessed out of hospital cardiac arrest. He was down for 7 minutes before EMS arrived. The Patient was found to be in PEA arrest, so CPR process was started. He was intubated and brought to the Emergency Department (ED) at our institution.

\subsection{Emergency department course}

In the ED, the patient was found to be once again in PEA arrest. Resuscitation process was continued, and he had a return of normal circulation but with low blood pressure requiring vasopressors. After resuscitation, he was noted to have a pulse of 58 per minute and a blood pressure of 130/85 on pressors.

\section{On exam:}

The patient was intubated, nonresponsive with a GCS of 3. He had a clear lung exam and normal cardiac exam with normal S1 and S2, no rubs, murmurs or gallops; and no neck veins distension was appreciated.

\section{Laboratory Data:}

WBC: 15.2. HG: 15.8 g/dl. HCT: $47.7 \%$. Plt: 186.

Na: 138 mEq/L. K: 3.8 mEq/L. Chloride: 101 mEq/L. Co2: 15 mEq/L. BUN: 16 mg/dl. Creatinine: 1.2 mg/dl. Glucose: 200 mg/dl. Anion Gap: $22 \mathrm{mEq} / \mathrm{L}$. Ca: 9 mg/dl. Mg: $2.2 \mathrm{mg} / \mathrm{dl}$.

CK: 647 U/L. CKMB: 15.3 IU/L with negative index. Troponin I: 0.71ng/ml.

ABGs: PH: 7.24. pco $^{2}:$ 32. HCO3: 13. $\mathrm{pO}^{2}:$ 144. Lactic Acid per ABG: 12.3.

POC Myoglobin: 190 ng/ml. POC BNP: 1080 pg/ml.

\section{ECG:}

The patient ECG from a previous ED visit showed only sinus tachycardia (see Figure 1).

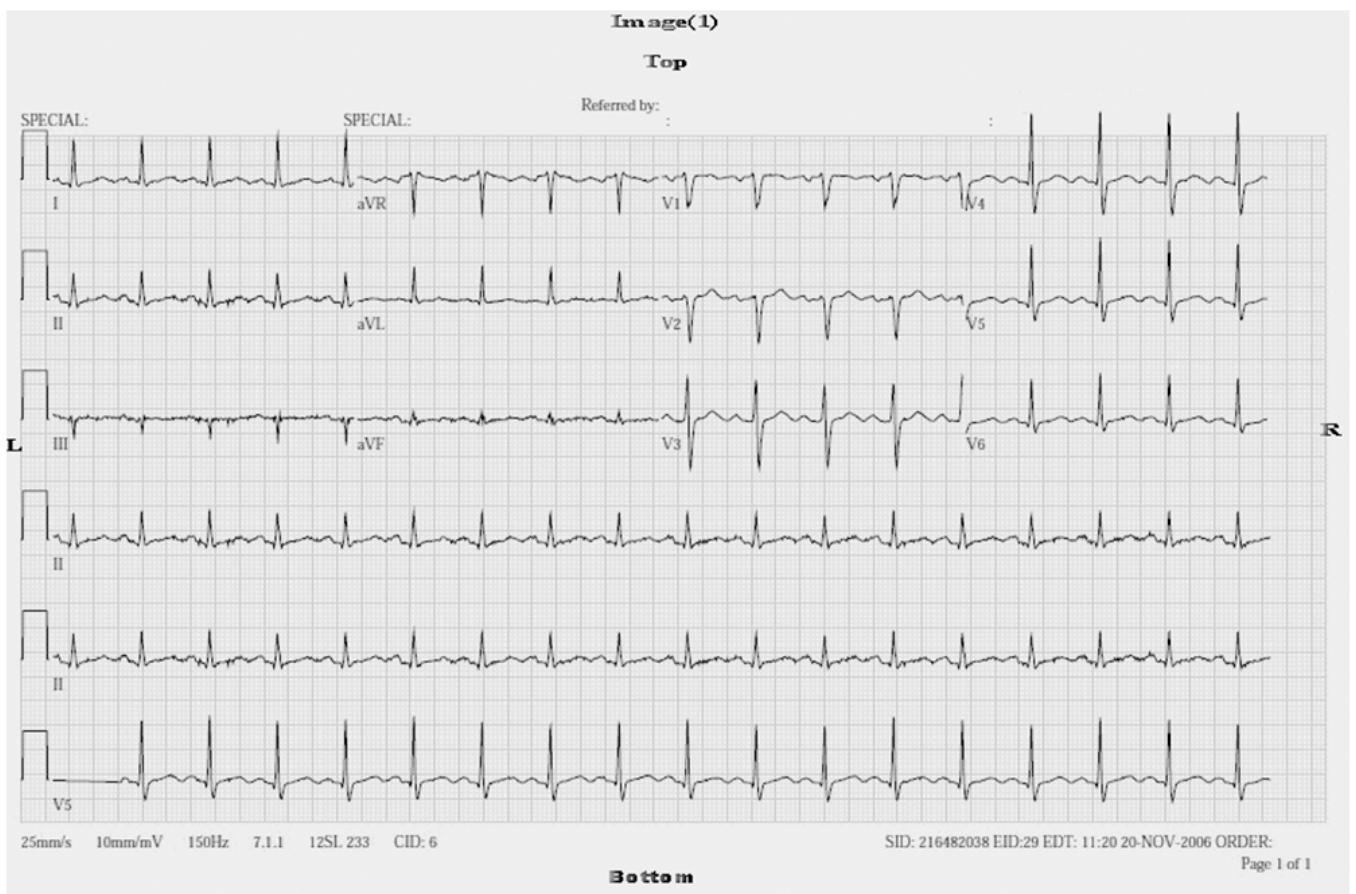

Figure 1. The patient base line ECG from a previous ED visit 
His ECG now showed: Severe sinus bradycardia with junctional escape rhythm, complete RBBB, Left anterior fascicular block evidenced by left axis deviation and ST-segment elevation in the inferior leads (see Figure 2).

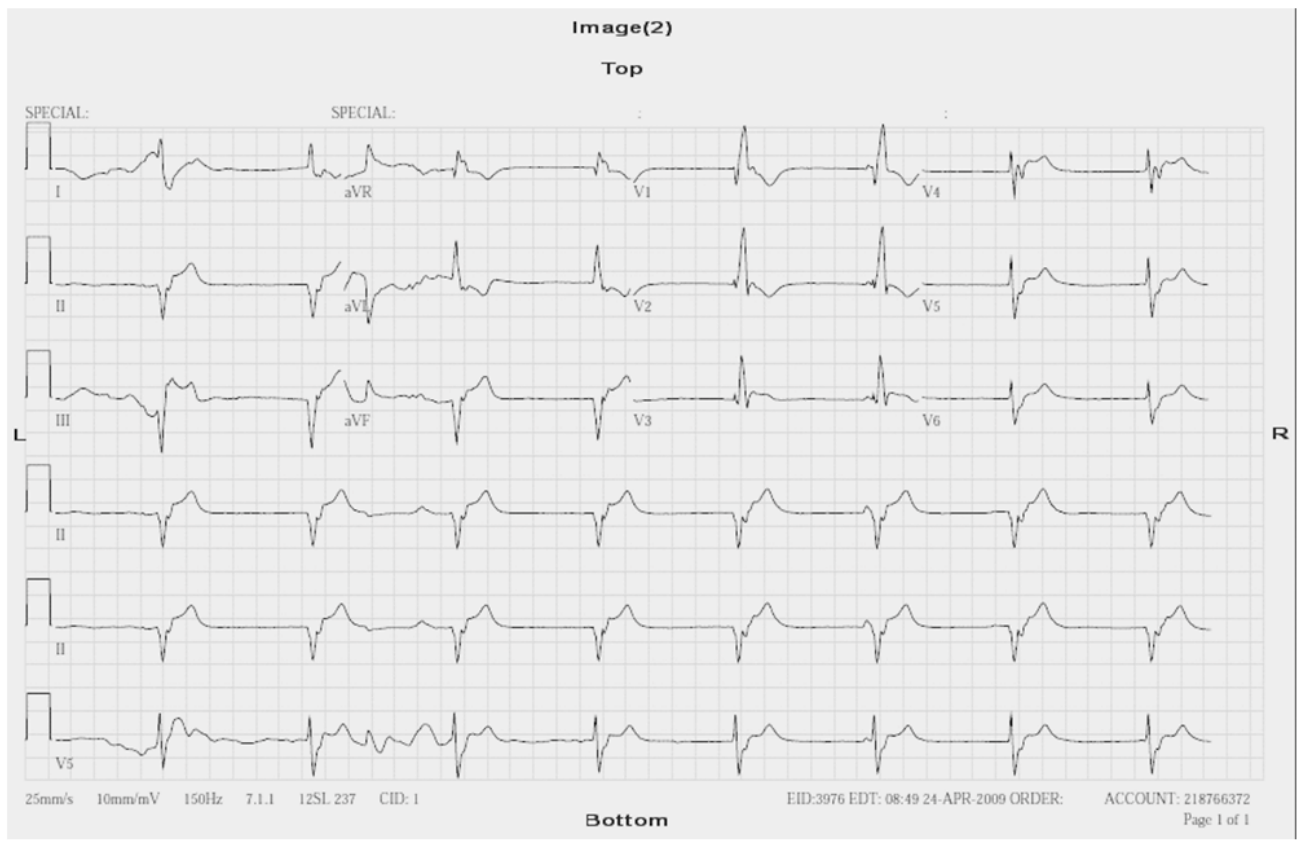

Figure 2. The patient ECG just after resuscitation

Given the patient's presentation, lab results and ECG findings, the patient was taken to the cath lab with a possible diagnosis of inferior wall ST-elevation MI.

\subsection{Cath lab results}

Coronary angiography revealed no obstructive lesions in the patient's coronary arteries.

The left main was completely normal. The circumflex showed mild luminal irregularities. The LAD showed diffuse disease up to $50 \%$ in the proximal portion and 30\% in the mid-distal portion (see Figure 3 and 4).

Figure 3. No obstructive lesions on the angiogram

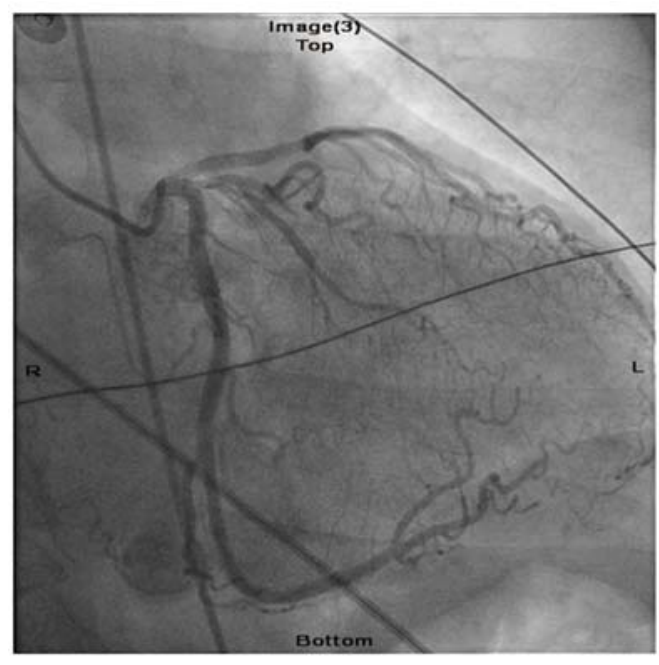


The RCA had only mild luminal irregularities with TIMI 3 flow (see Figure 5).

Figure 4. No obstructive lesions on the angiogram

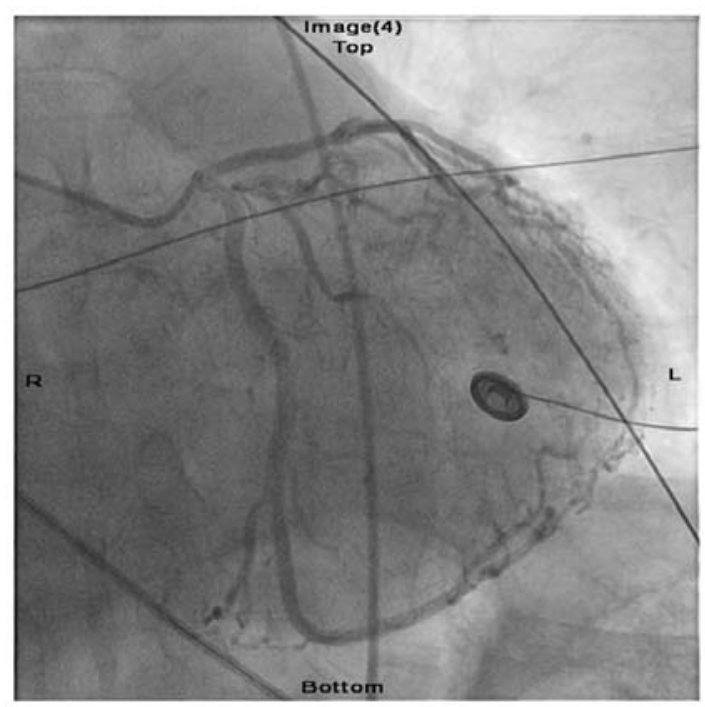

Figure 5. No obstructive lesions or filling defects in the right coronary artery.

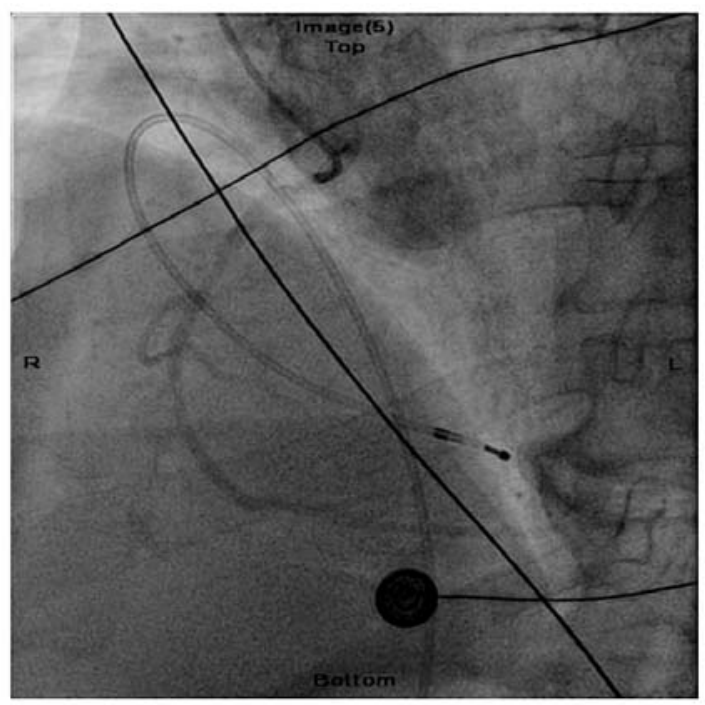

In the interim an intraaortic balloon pump was inserted in the right common femoral artery for hemodynamic support, and a transvenous pacemaker was placed from the femoral vein into the RV. Urgent trans-thoracic echo was performed which showed a Left ventricle estimated ejection fraction in the range of $65 \%$ to $70 \%$. Wall motion was normal; the cavity of the right ventricle was severely dilated, Wall thickness was normal and systolic function was severely reduced. The Right atrium was severely dilated (see Figure 6).

Given his clinical presentation, the ultrasound results and the Coronary angiography findings it was felt that the patient may be suffering from a massive PE.

Pulmonary Artery angiography was performed which showed bilateral pulmonary embolism more significant on the left branch of the pulmonary artery (see Figure 7). 
Mechanical thrombectomy using angiojet to the left inferior branch of the pulmonary artery was performed and local TPA infusion was delivered with $50 \%$ reduction of clot size.

Figure 6. Echocardiogram showing severe right ventricular dilation

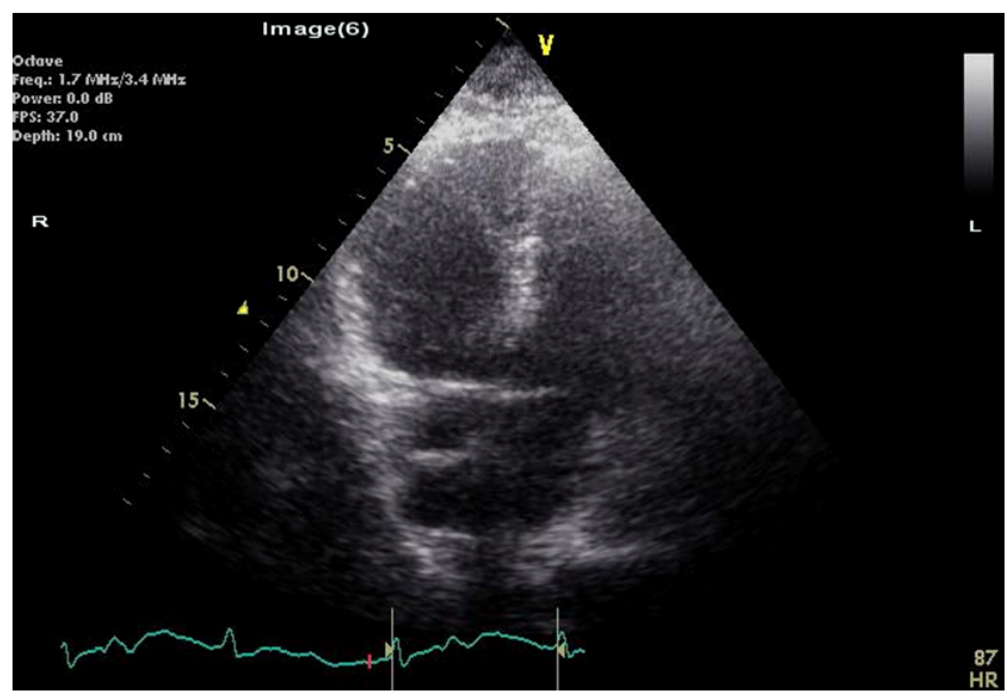

Figure 7. Significant emboli obstructing

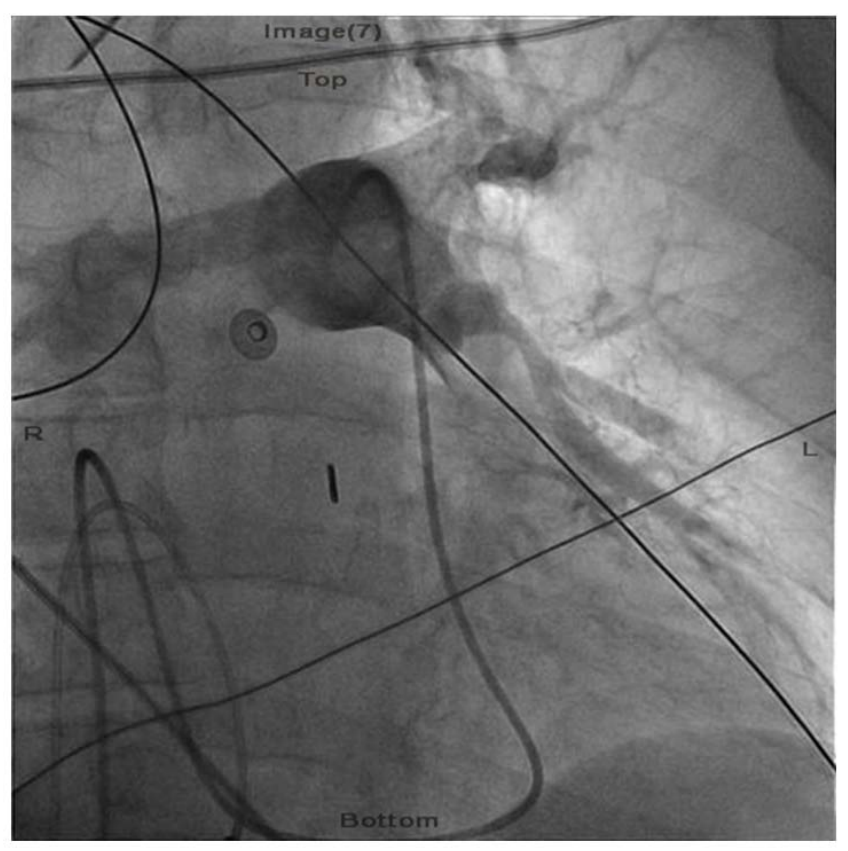
the left branches of the Pulmonary Artery

The patient significantly improved after the procedure with stable blood pressure and improvement in his oxygen saturation.

After the procedure, the patient was admitted to the cardiac intensive care unit. Following ECGs done after the procedure revealed normal sinus rhythm, normal conduction with resolution of the right bundle branch block and the left anterior fascicular blood in addition to the resolution of the ST-segment elevation (see Figure 8).

The peak troponin after his admission was 2.0. The patient had a complicated hospital course with severe anoxic brain injury and brain death two days later. 


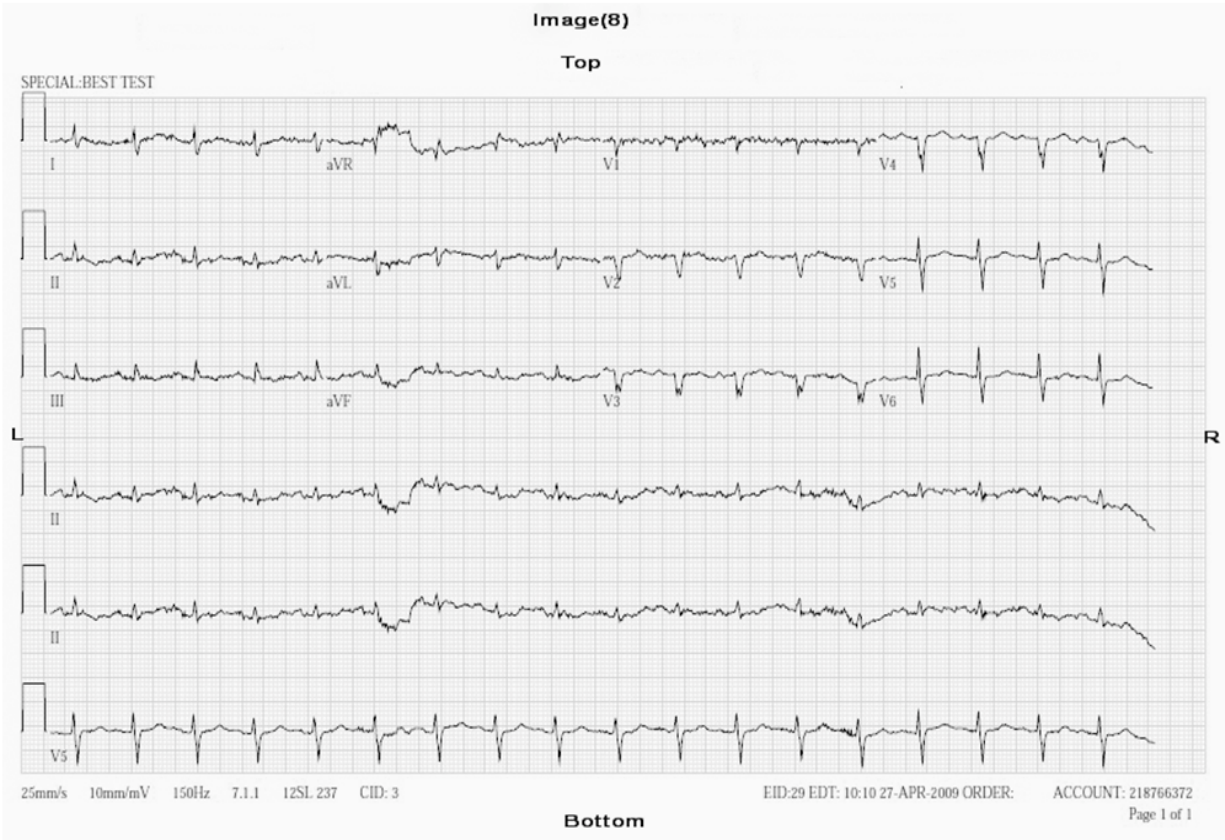

Figure 8. The patient ECG after the angiogram

\section{Case discussion}

Massive PE is a fatal condition with rapid deterioration and hemodynamic collapse. It consists of shock defined as a systolic blood pressure below 90 or a pressure drop of more than $40 \mathrm{mmHg}$ for at least 30 minutes not caused by arrhythmia, hypovolemia or sepsis. It is usually associated with severe hypoxia and acidosis secondary to the sudden drop in cardiac output.

Pulmonary embolism is associated with many ECG findings, most of which are non-specific and most can be explained by the sudden severe increase in the right ventricular afterload leading to dysfunction, hypoperfusion, dilation and in rare very severe cases to ischemic injury.

The typical and atypical ECG findings associated by PE include ${ }^{[1]}$ :

- Approximately one third of the patients have a normal ECG without any abnormal findings.

- Common ECG findings: Sinus tachycardia (The most common finding), non-specific ST segment and T wave changes especially in the right precordial leads, right axis deviation, complete or incomplete RBBB and the S1Q3T3 pattern.

- Less common findings: First degree heart block, atria tachyarrhythmia including atrial fibrillation, displacement of the transitional point to the left, long R wave in V1, left axis deviation (rare) and P "pulmonale"

Many case reports described patients presenting with massive pulmonary embolism and very rare atypical ECG findings especially ST-segment elevation in the anteroseptal leads (V1-V4). These studies proposed many hypotheses to explain the pathophysiology underlying these findings.

Budavary et al ${ }^{[2]}$ reported a case of simultaneous pulmonary embolism and acute myocardial infarction secondary to a previously undiagnosed patent foramen ovale (PFO). Paradoxical emboli crossing the PFO to the coronary arteries caused 
the acute myocardial infarction in this case. In our case, the Ultrasound didn't detect any septal defects that might explain the coronary embolization theory. Furthermore, there was no evidence of coronary obstruction on the coronary angiography.

Falterman et al ${ }^{[3]}$ described a case of massive PE with ST-elevation in V1-V3. In this case however, the other typical findings mentioned above were noted on the ECG (right axis and incomplete RBBB).

Another case reported by Livatidis and colleagues showed ST-elevation in the anteroseptal leads with normal axis and ST-depression in V6. In studies, severe right ventricular strain and hypoperfusion leading to ischemia was the suggested explanation.

Jia-Feng Lin and Colleagues ${ }^{[4]}$ reported a case about a 35-year-old male with massive PE and ST-elevation in V1-V4. In this study they discussed a combination of hemodynamic, anatomic, metabolic and autonomic effects of acute PE. They focused on the role of the severe right ventricular dilation and the increase in wall tension along with decrease in coronary flow caused by the sudden drop in cardiac output.

Matt Sampson, and C. Edward Rose, Jr, ${ }^{[5]}$, reported a case of a 22-year-old male with inferior leads ST-segment elevation in the setting of left lung atelectasis which completely resolved with the lung expansion. No clear explanation was provided for this ECG finding in their report.

1) To our knowledge, this is the first case of ST-elevation in the inferior leads in the setting of a massive PE.

Although the full explanation to this finding is still unclear we can emphasize some of the points that might explain this very atypical presentation:

2) The most likely explanation to the case is that the associated cardiac injury is multifactorial. Severe right ventricular dilation with significant increase in wall tension and oxygen consumption, sudden coronary hypoperfusion caused by the sudden drop in the right and left ventricular output, hypoxia caused by the massive $\mathrm{PE}$ and finally possible coronary spasm caused by hypoxia and increased right heart pressure might all have contributed to inducing the acute right ventricular ischemia which showed as ST-segment elevation in the inferior leads and an elevation in cardiac enzymes.

3) In our patient, again, The Echocardiogram didn't show any atrial septal defect and the angiogram images didn't show any filling defects in the RCA territory which makes RCA embolism very unlikely.

4) The massive PE was more prominent on the left side which might have caused a severe left lung atelectasis and ST-segment elevation similar to the case report mentioned above.

\section{Conclusion}

The most likely explanation to the ECG findings of ST-segment elevation in the inferior leads in the setting of pulmonary embolism is multifactorial. Severe right ventricular dilation with significant increase in wall tension and oxygen consumption, sudden coronary hypoperfusion caused by the sudden drop in the right and left ventricular output, hypoxia caused by the massive PE and finally possible coronary spasm caused by hypoxia and increased right heart pressure might all have contributed to inducing the acute right ventricular ischemia which showed as ST-segment elevation in the inferior leads and an elevation in cardiac enzymes.

\section{Patient consent section}

Written informed consent was obtained from the patient's next of kin for publication of this case report and accompanying images. A copy of the written consent is available for review by the Editor-in-Chief of this journal. 


\section{Conflict of interest disclosures}

The authors declare that they have no competing interests.

\section{Authors contributions}

TH: The director of the Catheterization labs. He was the attending physician on the case and provided the introduction section and reviewed the work before submitting.

MA: The cardiology fellow. Helped with the references of the case and had most of the contact with the patient.

SA: The first author. Wrote the whole manuscript and the discussion section. Organized the work and put the case together.

\section{References}

[1] Chou T, Knilans TK. Electrocardiography in clinical practice adult and pediatric. 4th ed. Philadelphia: Saunders. 1996; 167-70.

[2] Budavari AI, Glenn TJ, Will KK, Askew JW, Fortuin FD. A case of simultaneous pulmonary embolism and acute myocardial infarction secondary to a previously undiagnosed patent foramen ovale. J Hosp Med. 2009 May; 4(5): E5-9. PMid:19504585 http://dx.doi.org/10.1002/jhm.464

[3] Falterman TJ, Martinez JA, Daberkow D, et al. Pulmonary embolism with ST segment elevation in leads V1 to V4: case report and review of the literature regarding electrocardiographic changes in acute pulmonary embolism. J Emerg Med. 2001 ; 3: 255. http://dx.doi.org/10.1016/S0736-4679(01)00381-X

[4] Jia-Feng Lin, Yue-Chun Li, Peng-Lin Yang. A Case of Massive Pulmonary Embolism With ST Elevation in Leads V(1-4). Circ J. 2009; 73: 1157-1159. PMid:19096187 http://dx.doi.org/10.1253/circj.CJ-08-0474

[5] Sampson M, Rose CE Jr. Reversible ST-segment elevation associated with atelectasis of the left lung. South Med J. 2005 Sep;98(9):950-2. PMid:16217995 http://dx.doi.org/10.1097/01.smj.0000170726.78243.c1

[6] Shewan LG and Coats AJ. Ethics in the authorship and publishing of scientific articles Int J Cardiol. 2010 ; $144:$ 1-2. http://dx.doi.org/10.1016/j.ijcard.2010.07.030 